\title{
Caregiver Experiences of Discrimination and African American Adolescents' Psychological Health Over Time
}

\author{
Kahlil R. Ford \\ New York University
}

\author{
Noelle M. Hurd \\ University of Virginia
}

\author{
Robert J. Jagers and \\ Robert M. Sellers \\ University of Michigan
}

\begin{abstract}
The present study examined the effect of caregivers' experiences of racial discrimination on their adolescent children's psychological functioning among a sample of 264 African American dyads. Potential relations between caregiver discrimination experiences and a number of indicators of adolescents' (aged 12-17) psychological functioning over time were examined. It was found that caregiver discrimination experiences were positively related to adolescents' symptoms of depression and negatively related to their psychological wellbeing. Additional analysis revealed interactions between the effects of caregiver discrimination experiences and family income on all 3 outcomes. Greater caregiver discrimination experiences and lower family income were risk factors for the youth in the sample. These findings underscore the deleterious consequence of caregivers' discrimination experiences on African American youth's psychological health.
\end{abstract}

A growing body of research has demonstrated links between experiences with discrimination and adverse mental health outcomes among African Americans (Broman, 1997; Fisher, Wallace, \& Fenton, 2000; Jackson et al., 1996; Kessler, Mickelson, \& Williams, 1999; Williams, Neighbors, \& Jackson, 2008). Researchers have found that African American adolescents report experiencing educational, institutional, and peer discrimination (Fisher et al., 2000; Rosenbloom \& Way, 2004; Seaton, Caldwell, Sellers, \& Jackson, 2008; Seaton, Yip, \& Sellers, 2009) and that experiences with racial discrimination have been associated with lower self-esteem and greater symptoms of depression over time (Brody et al., 2006; Greene, Way, \& Pahl, 2006; Wong, Eccles, \& Sameroff, 2003). Coll et al. (1996) advanced an integrative theoretical model wherein ethnic minority youth's social positioning in society is acknowledged and assumed to exert substantial influence on their psychosocial development. Specifically, Coll et al. propose that social position fac-

This research was supported through a grant from the National Institute of Mental Health (NIMH5 R01 MH61967-02). The fourth author is the principal investigator of the grant. We thank the members of the African American Family Project for their help with data collection.

Correspondence concerning this article should be addressed to Kahlil R. Ford, c/o the BERC Group 22232 17th Ave SE, Suite 305, Bothell, WA 98021. Electronic mail may be sent to kahlil@ bercgroup.com. tors such as race, ethnicity, social class, and gender affect ethnic minority youth's developmental outcomes via social mechanisms such as discrimination. Furthermore, these pervasive and oppressive social mechanisms (e.g., discrimination) may operate through any number of processes to directly or indirectly shape minority youth's outcomes.

In their model, Coll et al. (1996) highlight the ways in which environments shaped by oppressive structures have the potential to affect ethnic minority youth's outcomes by altering family processes. Informed by this model, researchers have begun to investigate how caregivers' experiences with discrimination may influence the psychosocial outcomes of ethnic minority youth (Caughy, O'Campo, \& Muntaner, 2004; Gibbons, Gerrard, Cleveland, Wills, \& Brody, 2004; Johnson, 2001; Riina \& McHale, 2010). Thus, researchers have moved beyond individual-level analyses of the effects of perceived discrimination on psychological and physical well-being and have begun to study the ways in which adult caregivers' experiences with discrimination may negatively influence child outcomes. In the current study, we investigated how African American caregivers' experiences with

Child Development (C) 2012 Society for Research in Child Development, Inc. All rights reserved. 0009-3920/2013/8402-0009

DOI: $10.1111 /$ j.1467-8624.2012.01864.x 
discrimination shape their adolescent children's mental health outcomes. In addition, we assessed whether or not demographic variables, such as child gender and family income, moderated the association between caregivers' experiences with discrimination and adolescents' psychological outcomes. Finally, we considered whether or not these associations varied as youth progressed through different stages of adolescence.

Caregiver experiences with discrimination may influence adolescents' mental health outcomes through a variety of mechanisms. Researchers have found that witnessing acts of racial discrimination against other African Americans in youth's communities may contribute to increased depressive symptoms among youth, even after accounting for youth's personal experiences with discrimination (Simons et al., 2002). Consistent with this finding, witnessing discriminatory acts against their parents or caregivers may be particularly distressing for African American adolescents. Yet, it also is possible that caregivers' experiences with discrimination may influence adolescents' outcomes even when adolescents do not personally witness the discriminatory acts against their caregivers. As indicated by the stress model of discrimination (Clark, Anderson, Clark, \& Williams, 1999; Meyer, 2003), the experience of discrimination may negatively impact caregivers' health and well-being. Racially discriminatory experiences may impinge upon caregivers' parenting abilities by increasing stress, contributing to psychological distress or physical health problems, or increasing demands on caregivers' cognitive load. Thus, African American adolescents may be more vulnerable to mental health problems and experience lower psychologically well-being as a result of their caregivers' experiences with racial discrimination, even when adolescents do not personally witness the discriminatory acts.

Gibbons et al. (2004) investigated the potential effects of African American parents' perceived discrimination on child outcomes. They found that African American parents' discrimination experiences directly predicted their 10- to 12-year-old children's psychological distress and indirectly predicted their children's substance use over time via parent and child psychological distress. Riina and McHale (2010) found evidence that parental experiences of discrimination are detrimental to the quality of both parent-child and marital relationships among their sample of African American middle-class families. Specifically, they found that parents' experiences with discrimination predicted parentadolescent conflict, coparenting conflict, marital conflict, and parental warmth toward their adolescent children. In addition, they found that parental expressiveness and instrumentality moderated the effects of parents' discrimination on family relationship quality. In contrast, Caughy et al. (2004) did not find an association between African American parents' reports of personal experiences with racism and rates of behavior problems among their preschool-aged children. Caughy et al., however, found that of the parents who reported personal experiences with racism, those who reported active behavioral responses to racism reported that their children had fewer symptoms of depression and anxiety in comparison to the children of parents who reported fewer or no active behavioral responses to experiences with racism. It is clear that more research that directly investigates the possible link between parental experiences with racial discrimination and child outcomes is needed before any definitive conclusions can be drawn.

In addition, existing research evidence suggests that adolescence may be a key developmental period to explore the association between parental experiences with racial discrimination and African American child outcomes (Gibbons et al., 2004; Riina \& McHale, 2010). The inconsistency among the findings in previous research on racial discrimination and child outcomes may stem from the age differences of children in the study. The studies that found a link focused on adolescents as opposed to younger children. It is possible that the younger children did not possess the cognitive and social understanding necessary to perceive or process the meaning of racial discrimination (Aboud, 1989; Cross \& Fhagen-Smith, 2001; Quintana, 1998). Also, parents may be less likely to discuss issues related to race, including their own experiences with racial discrimination, with their younger children as opposed to their older children (Hughes \& Chen, 1997; McHale et al., 2006).

Even with a focus on adolescence as a developmental period, there also may be important age differences in the ways in which caregivers' experiences with racial discrimination may influence adolescents' mental health outcomes. For instance, the development of autonomy over time may be a key variable. Whereas, younger adolescents may be more strongly influenced by their caregivers' experiences with discrimination, older adolescents might be less sensitive to parental influence as they become more autonomous (Arnett, 1999). In addition, younger adolescents may have fewer coping resources than older adolescents and, as a consequence, be poorly equipped to cope with their 
caregivers' experiences with discrimination or the negative effects these experiences may have on their caregivers' parental functioning.

In addition to age, other demographic factors such as gender and family income may be important moderators of the potential association between caregivers' experiences with racial discrimination and child mental health outcomes. For instance, research on the effects of Black adolescents' experiences with racial discrimination and their own psychological health indicate important gender differences (e.g., Seaton, Caldwell, Sellers, \& Jackson, 2010). Seaton et al. (2010) found that older Black female adolescents exhibited higher depressive symptoms and lower life satisfaction in the context of high levels of perceived discrimination compared to older Black male adolescents. Thus, it is quite possible that caregivers' experiences with discrimination may differentially affect adolescent girls versus boys. Given that girls are often socialized to be more sensitive to interpersonal factors, adolescent girls may be more vulnerable to the negative effects of intrafamilial stress than adolescent boys (Gore, Aseltine, \& Colten, 1993). Furthermore, adolescent girls may experience the family setting as a more salient context than adolescent boys and, as a result, be more affected by their caregivers' experiences with racial discrimination. Moreover, adolescent girls may be more influenced by their interactions with their parents than adolescent boys (Gore et al., 1993), which again suggests that adolescent girls may be more negatively affected by their caregivers' experiences with discrimination than adolescent boys.

Family income also may exert an influence on the association between caregivers' discrimination experiences and adolescents' mental health outcomes. Researchers have noted that African Americans with higher socioeconomic statuses may experience more discrimination due to more frequent contact with Whites (Kessler et al., 1999). Yet, few researchers have investigated the potential of greater economic resources to help buffer against the negative psychological effects of exposure to discrimination. It is possible, for example, that African American adolescents with higher family income levels may be less negatively affected by their caregivers' discrimination experiences. African American adolescents with greater family incomes may experience fewer stressors in comparison to their lower income peers and thus may be less psychologically vulnerable. Furthermore, African American adolescents with higher family incomes may have greater access to resources, such as psychotherapy, that could help them cope with psychologically distressing events. The same, of course, is true for African American parents or caregivers who have greater financial resources; these adults could be less negatively affected by experiences with discrimination and consequently experience fewer problems that may interfere with their parenting abilities. Conversely, given that researchers have found that African American families with greater incomes may have more frequent experiences with discrimination, African American caregivers and adolescents in these families may be more negatively affected by these experiences as a result of the cumulative impact of these experiences. Thus, it is possible that family income may buffer or exacerbate the negative effects of caregivers' experiences with discrimination on African American adolescents' psychological outcomes.

\section{Current Study}

In the current study, we sought to replicate and build upon previous research examining the relation between caregivers' experiences with discrimination and the mental health outcomes of their adolescent children. We attempted to address three questions. First, do African American caregivers' experiences with racial discrimination influence their adolescent children's mental health outcomes? Second, do demographic factors such as the child's gender and family income moderate the association between caregivers' experiences with racial discrimination and adolescent outcomes? Finally, do these associations vary as adolescents progress from early to middle adolescence? We explored these questions among a sample of African American adolescents and their primary caregivers. Outcome variables in the current study included adolescents' perceived stress, symptoms of depression, and psychological well-being. In addition to assessing potential moderation effects of gender and income, we also controlled for whether or not adolescents' caregivers were their mother. We did this in light of the literature suggesting that maternal influences may be stronger than paternal or other familial adult influences (Gore et al., 1993). Furthermore, given that participants entered our study at various ages, we controlled for participants' age during their first wave of participation in our longitudinal study.

We expected that caregivers' experiences of racial discrimination would be associated with increased perceived stress and depressive symptoms as well as decreased psychological well-being among African American adolescents. We hypothesized that 
adolescent girls would be more negatively affected by their primary caregivers' discrimination experiences in comparison to adolescent boys. Given that higher familial income could serve as a risk or protective factor against the negative effects of caregivers' experiences with discrimination on adolescents' psychological outcomes, we did not have an a priori hypothesis regarding the potential moderating effects of familial income. Finally, we hypothesized that the association between primary caregivers' experiences with racial discrimination and adolescents' mental health outcomes would be strongest during early adolescence and become less strong as youth move from early adolescence through middle adolescence.

\section{Method}

\section{Participants}

The current study utilized data from the first two cohorts of a three-cohort, 3-year sequential study of race and psychosocial adjustment in African American adolescents and their primary caregivers. Of the 308 child-caregiver dyads that completed the first wave of the study, we used the 264 dyads in which (a) both the caregiver and the child were African American and (b) both members of the dyad had complete data for each of the variables for at least two waves of the study. Our longitudinal analyses focus on the first two cohorts of the study because these participants had the opportunity to participate in at least two waves of data collection, whereas Cohort 3 only participated in one wave of data collection. The caregivers' ages ranged from 23 to 78 years with a mean age of 41.85 years $(S D=7.99)$.
A small minority of the caregivers in the sample had less than a high school diploma (6\%), 12\% received a high school diploma, $65 \%$ attended at least some college, and $16 \%$ completed a graduate degree (i.e., master's, PhD, JD, MD) as their highest level of educational attainment. Most of the caregivers were the adolescent participants' mothers $(78 \%)$, fathers $(10 \%)$, or grandmothers $(6 \%)$. Approximately, $42 \%$ of the caregivers were married, 35\% were single, $16 \%$ were divorced, and 5\% were separated. Fifty-seven percent of the adolescents in the present sample were girls. At Wave 1, the adolescents' ages ranged from 12 to 17 years with a mean age of 13.7 years $(S D=1.2)$. See Table 1 for means, standard deviations, and intercorrelations between study variables.

The city in which the study was conducted has a population of approximately 110,000 people and African Americans comprise roughly 9\% of the city population. The city's median income $(\$ 50,160)$ slightly exceeds the national average $(\$ 48,451$; U.S. Census Bureau, 2006). Eighteen percent of the school district's students were eligible for free or reduced lunch. Although the median annual family income for African Americans in the city surpasses the national average, there is significant variation across the income distribution within the sample. Within the present sample, caregiver-reported annual family incomes ranged from less than $\$ 10,000$ to more than $\$ 130,000$ with a median income of $\$ 30,000-\$ 39,000$.

\section{Procedure}

The school district provided initial contact information for African American students in Grades 7

Table 1

Zero-Order Correlations, Means, and Standard Deviations for Adolescent and Caregiver Variables

\begin{tabular}{|c|c|c|c|c|c|c|c|c|}
\hline Variable & 1 & 2 & 3 & 4 & 5 & 6 & 7 & 8 \\
\hline \multicolumn{9}{|l|}{ Adolescent } \\
\hline 1. Depressive symptoms & - & & & & & & & \\
\hline 2. Well-being & $-0.431^{* *}$ & - & & & & & & \\
\hline 3. Stress & $0.578^{* *}$ & $-0.450^{* *}$ & - & & & & & \\
\hline 4. Age & $-0.122^{* *}$ & $0.100^{*}$ & $-0.084^{*}$ & - & & & & \\
\hline 5. Gender & $-0.116^{* *}$ & $-0.096^{*}$ & $-0.180^{* *}$ & 0.038 & - & & & \\
\hline \multicolumn{9}{|l|}{ Caregiver } \\
\hline 6. Discrimination & $0.093^{*}$ & $-0.159^{* *}$ & 0.046 & -0.036 & 0.081 & - & & \\
\hline 7. Mother & 0.01 & 0 & -0.034 & $-0.137^{* *}$ & -0.023 & -0.02 & - & \\
\hline 8. Income & -0.047 & $0.098^{*}$ & $-0.106^{* *}$ & $0.121^{* *}$ & 0.009 & $-0.195^{* *}$ & $0.84^{* *}$ & - \\
\hline$M$ & 1.74 & 3.64 & 2.89 & 13.70 & 0.42 & 1.02 & 0.78 & 5.33 \\
\hline$S D$ & 0.47 & 0.51 & 0.48 & 1.21 & 0.49 & 0.79 & 0.42 & 3.88 \\
\hline
\end{tabular}

${ }^{*} p<.05 .{ }^{* *} p<.01$. 
through 11. Letters were sent to students' legal guardians requesting permission for students and their primary caregivers to participate in our study. If there was more than one eligible child per household, only one child was selected to participate in the study (selection was random). Follow-up calls were made to caregivers to obtain informed consent and to confirm that the child identified was African American. Once informed consent was obtained, an appointment was scheduled for a data collection session with the child. All data collection sessions took place after school. Data collection sessions with primary caregivers were conducted separately. Adolescents and primary caregivers were asked to complete surveys consisting of measures of racial identity, racial socialization, discrimination, and several indices of psychological adjustment. Surveys were administered in small groups administered by trained research assistants (primarily African American). These administrations took place in community settings (e.g., community centers, public libraries, local shopping centers). Participants were informed that all responses were confidential, that they could skip any item to which they did not wish to respond, and that they would not be penalized for withdrawing from the study at any point. The questionnaire took $60-90 \mathrm{~min}$ to be completed, on average. Compensation included a $\$ 20$ gift certificate to a local mall for adolescent participants and monetary compensation of $\$ 40$ for caregiver participants. Of the 742 households contacted to participate in the study, 546 children $(74 \%$ child response rate) and 366 primary caregivers (49\% caregiver response rate) participated across the life of the study.

\section{Measures}

Caregiver racial discrimination. Caregivers' experiences with discrimination during the past year were measured at each time point using the Racism and Life Experience Scales (RaLES). The RaLES (Time $1 \alpha=.94$, Time $2 \alpha=.91$, Time $3 \alpha=.90$ ) consists of 18 items measuring how often individuals were discriminated against because of their race (Harrell, 1997). A sample item includes: "In the past year, how often have you been ignored, overlooked, or not given service (in a restaurant, store, etc.) because of your race?" Caregivers were asked to respond on a 6-point scale regarding the frequency with which they experienced the event $(0=$ never to $5=$ once a week or more $)$. Higher scores indicate more experiences with discrimination. The RaLES has been found to be a reliable and valid measure of perceived discrimination (Harrell, 1997).

Depressive symptoms. The Center for Epidemiological Studies-Depression Scale was used to assess the presence and frequency of symptoms associated with depression (Radloff, 1977) at all three time points. The 20-item inventory (Time $1 \alpha=.85$, Time $2 \alpha=.85$, Time $3 \alpha=.87$ ) allowed participants to rate the frequency of specific feelings such as loneliness in the past week, ranging from 1 = rarely or none of the time $(<1$ day) to $4=$ most or all of the time (5-7 days). Thus, higher scores indicated more experiences of depressive symptoms. Sample items include: "I was bothered by things that do not usually bother me" and "I felt depressed." Scores were averaged across the 20 items.

Well-being. Adolescents' psychological well-being was measured at all three time points using a 24-item shortened version of the Psychological Well-Being Scale (Ryff, 1989). This shortened version of the Psychological Well-Being Scale (Time $1 \alpha=.84$, Time $2 \alpha=.84$, Time $3 \alpha=.85$ ) was used to assess participants' level of well-being along six dimensions: self-acceptance, positive relations with others, autonomy, environmental mastery, purpose in life, and personal growth. Participants indicated their agreement with a series of items using a 5-point Likert-type scale ranging from $1=$ strongly disagree to $5=$ strongly agree. Sample items include: "In general, I feel I am in charge of my life" and "In general I feel confident and positive about myself." The Well-Being Scale score was derived by averaging the scores from all 24 items. Higher scores on the composite scale indicated higher levels of psychological well-being.

Perceived Stress Scale. Participants' perceived stress was measured at each time point using the Perceived Stress Scale (Cohen, Kamarck, \& Mermelstein, 1983). The Perceived Stress Scale (Time $1 \alpha=.65$, Time $2 \alpha=.66$, Time $3 \alpha=.67$ ) consists of 14 items that ask how often in the last month individuals have experienced symptoms of stress. Sample items include feeling nervous and being upset because of something that happened unexpectedly. Response options ranged from $0=$ never to $4=$ very often . Scores were averaged across the 14 items; higher scores on the scale indicate more perceived stress.

\section{Results}

We used hierarchical linear modeling to examine the associations between caregivers' experiences with discrimination and adolescents' depressive symptoms, well-being, and perceived stress over 
time. Multilevel models have been used frequently to study individual growth (Raudenbush \& Bryk, 2002; Hox, 2002). They provide many distinct advantages for studying trajectories of outcomes over time, including the ability to describe change at both the within- and between-person levels, the ability to test many functional forms of change, and the ability to handle data that are both incomplete and unequally spaced in time. Multilevel models can also be used to combine data from multiple cohorts to define a single growth curve (e.g., Miyazaki \& Raudenbush, 2000; Raudenbush \& Chan, 1992). These specialized models enable researchers to test the tenability of the assumption that data from distinct but overlapping age cohorts can be combined to accurately approximate the developmental trajectory of a single cohort within a true longitudinal design (Bell, 1953, 1954; McArdle, Ferrer-Caja, Hamagami, \& Woodcock, 2002). This class of models is known as a linkage model (Miyazaki \& Raudenbush, 2000). In a linkage model, an analyst compares a full model, including the effect of cohort, with a restricted model, which does not include the effect of cohort. The two models are compared using deviance statistics. If the deviance statistics for the full and restricted models differ significantly $\left(\chi_{\text {critical }}^{2} p<.05\right)$, the cohort effects are retained in subsequent analysis. If the deviance statistics do not differ significantly $\left(\chi_{\text {critical }}^{2} p>.05\right)$, the data have achieved convergence, meaning that the trajectory of the outcome variable is indistinguishable for each cohort. The cohort effects are then excluded from further analyses.

In the present study, we tested linkage models to ensure that data from Cohorts 1 and 2 of the study could be combined to approximate trajectories of depressive symptoms $\left(\chi_{\text {critical }}^{2}=8.24, p=.08\right)$, perceived stress $\left(\chi_{\text {critical }}^{2}=2.46, p>.500\right)$, and wellbeing $\left(\chi_{\text {critical }}^{2}=2.94, p>.500\right)$. These tests showed that these data could be combined to approximate a single trajectory for each aspect of adolescent mental health, describing the development of these variables over the ages of 12-17 years. Figure 1 shows the plot of the mean values for each of the mental health variables over time, along with a plot of caregiver's discrimination experiences.

\section{Question 1}

This question examined the relation between caregivers' discrimination experiences and adolescent mental health over time. We tested the first research question using the following equation.

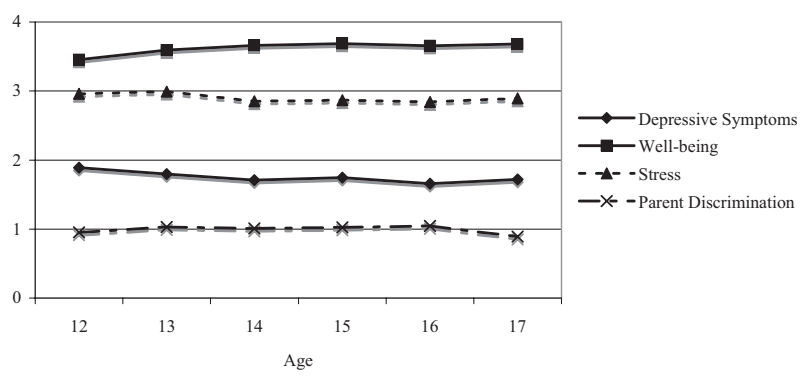

Figure 1. Caregiver discrimination, child well-being, child stress, and child depressive symptoms over time.

$$
\begin{aligned}
\text { Mental Health }_{i t}= & \pi_{0 i}(\text { Intercept })+\pi_{1 i}(\text { Linear Trend }) \\
& +\pi_{2 i}(\text { Caregiver Discrimination })+u
\end{aligned}
$$

where $\pi_{1 i}$ is a linear trend for each mental health variable, centered at age $13, \pi_{2 i}$ is a time-varying covariate representing the caregiver's perceived discrimination at a particular measurement occasion, and $u$ is an error term. Because the average age of our sample was around 13 years old, the data were centered at age 13 . Thus, the intercept represents the value of each mental health variable at age 13 . Results of these analyses for each outcome variable (i.e., depressive symptoms, perceived stress, and psychological well-being) are summarized in the first column of Tables 2-4.

The linear trend for depressive symptoms indicated a statistically significant decline over time (column 1, Table 2). Additionally, caregivers' discrimination experiences and adolescents' symptoms of depression were positively correlated at the trend level. When caregivers reported more discrimination experiences, adolescents reported increased depressive symptoms. The results also indicated an overall increase in well-being across the sample (column 1, Table 3). Caregivers' perceived discrimination experiences were negatively related to adolescents' psychological well-being, such that increased caregiver perceptions of discrimination were related to lower adolescent ratings of well-being. Finally, adolescents' reports of perceived stress also declined over time. However, there was no relation between caregivers' discrimination experiences and adolescents' perceived stress (column 1, Table 2).

\section{Question 2}

The second research question examined whether or not demographic factors (i.e., adolescents' gender and family income) moderated the association between caregivers' experiences with racial discrimination and adolescent outcomes. We added gender and caregiver income to the intercept, the linear trend slope, 
Table 2

Fixed Effects for Depressive Symptoms

\begin{tabular}{|c|c|c|c|c|c|c|c|c|c|}
\hline \multirow[b]{2}{*}{ Variable } & \multicolumn{3}{|c|}{ Question 1} & \multicolumn{3}{|c|}{ Question 2} & \multicolumn{3}{|c|}{ Question 3} \\
\hline & $\beta$ & $S E$ & $d f$ & $\beta$ & $S E$ & $d f$ & $\beta$ & $S E$ & $d f$ \\
\hline Intercept (depressive symptoms), $\pi_{00}$ & 0.72 & 0.04 & $261^{* * *}$ & 1.70 & 0.05 & $257^{* * *}$ & 1.68 & 0.06 & $257^{* * *}$ \\
\hline Age at Wave $1, \beta_{01}$ & & & & 0.00 & 0.04 & 257 & -0.03 & 0.05 & 257 \\
\hline Male, $\beta_{02}$ & & & & -0.07 & 0.09 & 257 & -0.03 & 0.10 & 257 \\
\hline Mother, $\beta_{03}$ & & & & -0.01 & 0.11 & 257 & -0.15 & 0.13 & 257 \\
\hline Income, $\beta_{04}$ & & & & -0.01 & 0.01 & 257 & -0.02 & 0.01 & $257^{\dagger}$ \\
\hline Linear trend, $\pi_{10}$ & -0.04 & 0.01 & $261^{* *}$ & -0.04 & 0.02 & $257^{\dagger}$ & -0.04 & 0.02 & $257^{*}$ \\
\hline Age at Wave $1, \beta_{11}$ & & & & 0.01 & 0.01 & 257 & 0.01 & 0.01 & 257 \\
\hline Male, $\beta_{12}$ & & & & 0.02 & 0.03 & 257 & 0.02 & 0.03 & 257 \\
\hline Mother, $\beta_{13}$ & & & & -0.03 & 0.04 & 257 & -0.05 & 0.04 & 257 \\
\hline Income, $\beta_{14}$ & & & & 0.00 & 0.00 & 257 & 0.00 & 0.00 & 257 \\
\hline Caregiver discrimination, $\pi_{20}$ & 0.05 & 0.03 & $553^{\dagger}$ & $0.05^{\dagger}$ & 0.03 & $541^{\dagger}$ & 0.08 & 0.05 & 536 \\
\hline Age at Wave $1, \beta_{21}$ & & & & -0.03 & 0.02 & 541 & -0.01 & 0.04 & 536 \\
\hline Male, $\beta_{22}$ & & & & -0.07 & 0.05 & 541 & -0.10 & 0.07 & 536 \\
\hline Mother, $\beta_{23}$ & & & & 0.05 & 0.07 & 541 & 0.22 & 0.10 & $536^{*}$ \\
\hline Income, $\beta_{24}$ & & & & 0.00 & 0.01 & 541 & 0.02 & 0.01 & 536 \\
\hline Linear $\times$ Caregiver Discrimination, $\pi_{30}$ & & & & & & & -0.01 & 0.03 & 536 \\
\hline Age at Wave $1, \beta_{31}$ & & & & & & & -0.01 & 0.01 & 536 \\
\hline Male, $\beta_{32}$ & & & & & & & 0.04 & 0.03 & 536 \\
\hline Mother, $\beta_{33}$ & & & & & & & -0.10 & 0.05 & $536^{*}$ \\
\hline Income, $\beta_{34}$ & & & & & & & -0.01 & 0.00 & $536^{*}$ \\
\hline
\end{tabular}

${ }^{\dagger} p<.10 .{ }^{*} p<.05 .{ }^{* *} p<.01 .{ }^{* * *} p<.001$.

Table 3

Fixed Effects for Well-Being

\begin{tabular}{|c|c|c|c|c|c|c|c|c|c|}
\hline \multirow[b]{2}{*}{ Variable } & \multicolumn{3}{|c|}{ Question 1} & \multicolumn{3}{|c|}{ Question 2} & \multicolumn{3}{|c|}{ Question 3} \\
\hline & $\beta$ & $S E$ & $d f$ & $\beta$ & $S E$ & $d f$ & $\beta$ & $S E$ & $d f$ \\
\hline Intercept (well-being), $\pi_{00}$ & 3.68 & 0.05 & $261^{* * *}$ & 3.69 & 0.05 & $257^{* * *}$ & 3.67 & 0.07 & $257^{* * *}$ \\
\hline Age at Wave $1, \beta_{01}$ & & & & 0.00 & 0.05 & 257 & 0.00 & 0.06 & 257 \\
\hline Male, $\beta_{02}$ & & & & 0.05 & 0.09 & 257 & 0.01 & 0.10 & 257 \\
\hline Mother, $\beta_{03}$ & & & & -0.04 & 0.12 & 257 & 0.01 & 0.14 & 257 \\
\hline Income, $\beta_{04}$ & & & & 0.02 & 0.01 & $257^{\dagger}$ & 0.03 & 0.01 & $257^{* * *}$ \\
\hline Linear trend, $\pi_{10}$ & 0.04 & 0.02 & $555^{* *}$ & 0.04 & 0.02 & $543^{\dagger}$ & 0.04 & 0.02 & $538^{*}$ \\
\hline Age at Wave $1, \beta_{11}$ & & & & 0.00 & 0.01 & 543 & -0.01 & 0.01 & 538 \\
\hline Male, $\beta_{12}$ & & & & -0.05 & 0.03 & 543 & -0.04 & 0.03 & 538 \\
\hline Mother, $\beta_{13}$ & & & & 0.03 & 0.04 & 543 & 0.04 & 0.04 & 538 \\
\hline Income, $\beta_{14}$ & & & & 0.00 & 0.00 & 543 & 0.00 & 0.00 & 538 \\
\hline Caregiver discrimination, $\pi_{20}$ & -0.09 & 0.03 & $555^{* * *}$ & -0.08 & 0.03 & $543^{* *}$ & -0.07 & 0.06 & 538 \\
\hline Age at Wave $1, \beta_{21}$ & & & & 0.02 & 0.02 & 543 & 0.02 & 0.04 & 538 \\
\hline Male, $\beta_{22}$ & & & & -0.06 & 0.06 & 543 & -0.03 & 0.07 & 538 \\
\hline Mother, $\beta_{23}$ & & & & -0.02 & 0.08 & 543 & -0.08 & 0.11 & 538 \\
\hline Income, $\beta_{24}$ & & & & 0.00 & 0.01 & 543 & -0.02 & 0.01 & 538 \\
\hline Linear $\times$ Caregiver Discrimination, $\pi_{30}$ & & & & & & & -0.02 & 0.03 & $538^{\dagger}$ \\
\hline Age at Wave $1, \beta_{31}$ & & & & & & & 0.01 & 0.01 & 538 \\
\hline Male, $\beta_{32}$ & & & & & & & -0.03 & 0.04 & 538 \\
\hline Mother, $\beta_{33}$ & & & & & & & 0.03 & 0.05 & 538 \\
\hline Income, $\beta_{34}$ & & & & & & & 0.01 & 0.01 & $538^{*}$ \\
\hline
\end{tabular}

${ }^{\dagger} p<.10 .{ }^{*} p<.05 .{ }^{* *} p<.01 .{ }^{* * *} p<.001$. 
Table 4

Fixed Effects for Perceived Stress

\begin{tabular}{|c|c|c|c|c|c|c|c|c|c|}
\hline \multirow[b]{2}{*}{ Variable } & \multicolumn{3}{|c|}{ Question 1} & \multicolumn{3}{|c|}{ Question 2} & \multicolumn{3}{|c|}{ Question 3} \\
\hline & $\beta$ & $S E$ & $d f$ & $\beta$ & $S E$ & $d f$ & $\beta$ & $S E$ & $d f$ \\
\hline Intercept (stress), $\pi_{00}$ & 2.90 & 0.04 & $261^{* * *}$ & 2.87 & 0.05 & $257^{* * *}$ & 2.92 & 0.07 & $257^{* * *}$ \\
\hline Age at Wave $1, \beta_{01}$ & & & & -0.03 & 0.04 & 257 & 0.00 & 0.06 & 257 \\
\hline Male, $\beta_{02}$ & & & & -0.18 & 0.09 & $257^{*}$ & -0.10 & 0.10 & 257 \\
\hline Moilier, $\beta_{03}$ & & & & 0.03 & 0.11 & 257 & 0.02 & 0.13 & 257 \\
\hline Income, $\beta_{04}$ & & & & -0.02 & 0.01 & $257^{*}$ & -0.04 & 0.01 & $257^{* * *}$ \\
\hline Linear trend, $\pi_{10}$ & -0.03 & 0.01 & $555^{*}$ & -0.02 & 0.02 & 543 & -0.03 & 0.02 & 538 \\
\hline Age at Wave $1, \beta_{11}$ & & & & 0.02 & 0.01 & 543 & 0.02 & 0.01 & $538^{\dagger}$ \\
\hline Male, $\beta_{12}$ & & & & 0.01 & 0.03 & 543 & 0.00 & 0.03 & 538 \\
\hline Mother, $\beta_{13}$ & & & & 0.00 & 0.04 & 543 & -0.01 & 0.04 & 538 \\
\hline Income, $\beta_{14}$ & & & & 0.00 & 0.00 & 543 & 0.00 & 0.00 & 538 \\
\hline Caregiver discrimination, $\pi_{20}$ & 0.03 & 0.03 & 555 & 0.03 & 0.03 & 543 & -0.01 & 0.05 & 538 \\
\hline Age at Wave $1, \beta_{21}$ & & & & -0.02 & 0.02 & 543 & -0.05 & 0.04 & 538 \\
\hline Male, $\beta_{22}$ & & & & 0.01 & 0.05 & 543 & -0.05 & 0.07 & 538 \\
\hline Mother, $\beta_{23}$ & & & & -0.11 & 0.07 & 543 & -0.10 & 0.10 & 538 \\
\hline Income, $\beta_{24}$ & & & & 0.01 & 0.01 & 543 & 0.03 & 0.01 & $538^{* *}$ \\
\hline Linear $\times$ Caregiver Discrimination, $\pi_{30}$ & & & & & & & 0.04 & 0.03 & 538 \\
\hline Age at Wave $1, \beta_{31}$ & & & & & & & -0.01 & 0.01 & 538 \\
\hline Male, $\beta_{32}$ & & & & & & & 0.05 & 0.04 & 538 \\
\hline Mother, $\beta_{33}$ & & & & & & & 0.00 & 0.05 & 538 \\
\hline Income, $\beta_{34}$ & & & & & & & -0.01 & 0.01 & $538^{* *}$ \\
\hline
\end{tabular}

${ }^{\dagger} p<.10 .{ }^{*} p<.05 .{ }^{* *} p<.01 .{ }^{* * *} p<.001$.

and the caregiver discrimination slope to test this hypothesis. As mentioned previously, we also included participants' age at Wave 1 and caregivers' relationship to the target child (mother vs. nonmother). We included age at Wave 1 of the study to assess whether or not the age at which participants entered the study affected their initial status on the outcome variables. We also assessed whether participants' age at Wave 1 affected the relation between the independent variables and the outcome variables (Fitzmaurice, Laird, \& Ware, 2004). We used the following equations to test this research question.

$$
\begin{aligned}
& \left.\pi_{0 i} \text { (Intercept }\right)=\beta_{00}(\text { Intercept })+\beta_{01}(\text { Age at Wave } 1) \\
& +\beta_{02}(\text { Gender })+\beta_{03}(\text { Mother })+\beta_{04}(\text { Income })+u \\
& \left.\pi_{1 i} \text { (Linear Trend }\right)=\beta_{10}(\text { Intercept }) \\
& \left.+\beta_{11} \text { (Age at Wave } 1\right)+\beta_{12}(\text { Gender }) \\
& \left.+\beta_{13} \text { (Mother }\right)+\beta_{14}(\text { Income })
\end{aligned}
$$

None of these demographic variables moderated the relation between caregiver discrimination and depressive symptoms (column 2, Table 2), wellbeing (column 2, Table 3), or perceived stress (column 2, Table 4).

\section{Question 3}

Our third question examined whether or not the associations between caregivers' discrimination experiences and adolescents' psychological outcomes varied over time and whether demographic factors (participants' gender and family income) influenced these associations. We controlled for the relationship of the caregiver to the target child in these analyses. We used the following equations to test this research question.

$\pi_{2 i}($ Caregiver Discrimination $)=\beta_{20}$ (Intercept $)$

$=\beta_{21}$ (Age at Wave 1$)+\beta_{22}$ (Gender) $+\beta_{23}$ (Mother)

$+\beta_{24}$ (Income)

$\pi_{3 i}$ (Linear Trend $\times$ Caregiver Discrimination $)$

$=\beta_{30}($ Intercept $)+\beta_{31}$ (Age at Wave 1$)+\beta_{32}($ Gender $)$

$+\beta_{33}$ (Mother) $+\beta_{34}$ (Income)

We probed significant interactions in two ways. First, we investigated group differences in the slopes from the three-way interaction (Dawson \& Richter, 2006). We compared the following four groups: (a) caregivers reporting higher income $(+1$ 
$S D)$ and higher discrimination (+1 SD), (b) caregivers reporting higher income $(+1 S D)$ and lower discrimination $(-1 S D),(\mathrm{c})$ caregivers reporting lower income $(-1 S D)$ and higher discrimination $(+1 S D)$, and (d) caregivers reporting lower income ( $-1 S D)$ and lower discrimination $(-1 S D)$. Next, we examined the simple slopes for each group to assess whether they differed from zero (Preacher, Curran, \& Bauer, 2006). Our first set of slope tests assessed whether adolescent depressive symptoms, wellbeing, or stress changed from age 13 to 17 years in each of the four groups. This set of tests examined whether the interaction between income and caregiver discrimination differed over time for each group. A second set of slope tests examined between-group differences in the outcomes at ages 13 and 17, respectively. For example, these tests showed whether the higher income and higher discrimination group reported higher or lower depressive symptoms at age 13 years than the higher income-lower discrimination group.

Depressive symptoms. We found that caregivers' perceived discrimination experiences and income moderated the trajectory of adolescents' depressive symptoms (column 3, Table 2; Figure 2). The slope for adolescents of caregivers who reported higher discrimination and higher income differed from the slope for the caregivers who reported higher income and lower discrimination, $t=-2.10, p=.04$. At age 13 years, adolescents in the higher incomehigher discrimination group reported higher levels of depressive symptoms than adolescents in the higher income-lower discrimination group, $\omega=$ $.15(.06), t=2.48, p=.01$. By age 17, the higher income-higher discrimination group's depressive symptoms declined significantly, $\omega=-.08$ (.04), $t=-1.99, p=.05$. Adolescents in the higher incomelower discrimination group reported relatively low levels of depressive symptoms at age 13 years and maintained low levels until age 17, $\omega=.01(.03)$, $t=0.24, p=.81$. The simple slopes test also indicated a significant decline in depressive symptoms for the lower income-lower discrimination group, $\omega=-.07$ (.04), $t=-1.93, p=.05$. In contrast, adolescents in the lower income-higher discrimination group had relatively high levels of depressive symptoms and maintained high levels until age 17, $\omega=-.03(.04)$, $t=-0.71, p=.48$. The higher income-higher discrimination group reported a relatively high level of depressive symptoms at age 13, $\omega=.15(.07)$, $t=2.12, p=.03$, but also experienced the most precipitous decline over time.

Well-being. In addition, we found that caregiver discrimination and income moderated the trajectory of adolescent well-being (column 3, Table 3; Figure 3). We found differences between children of caregivers who reported relatively lower levels of discrimination, but differed in levels of income, $t=-2.48, p=.01$. At age 13 years, the lower income-lower discrimination group reported lower levels of well-being than the higher income-lower discrimination group. The lower income-lower discrimination group reported increased well-being at age 17, $\omega=.11(.04), t=2.73, p=.01$. By age 17, the two groups did not differ in levels of wellbeing, $\omega=.004, t=0.202, p=.83$. In sum, the lower income-lower discrimination group began the study with low levels of well-being relative to the higher income-lower discrimination group. The lower income-lower discrimination group reported higher well-being over time and the higher income-lower discrimination group maintained high levels of well-being over time.

Additional analyses explored whether or not the two income groups differed in well-being as a function of racial discrimination. At age 13 years, the higher

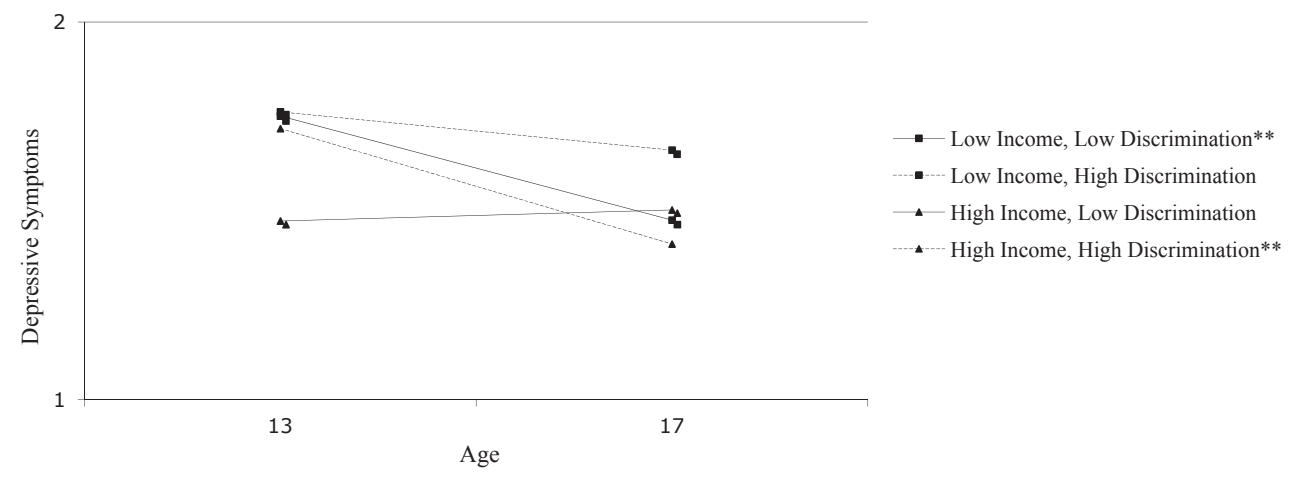

Figure 2. Three-way interaction between caregiver discrimination, income, and age on child depressive symptoms. ${ }^{* *} p<.01$. 


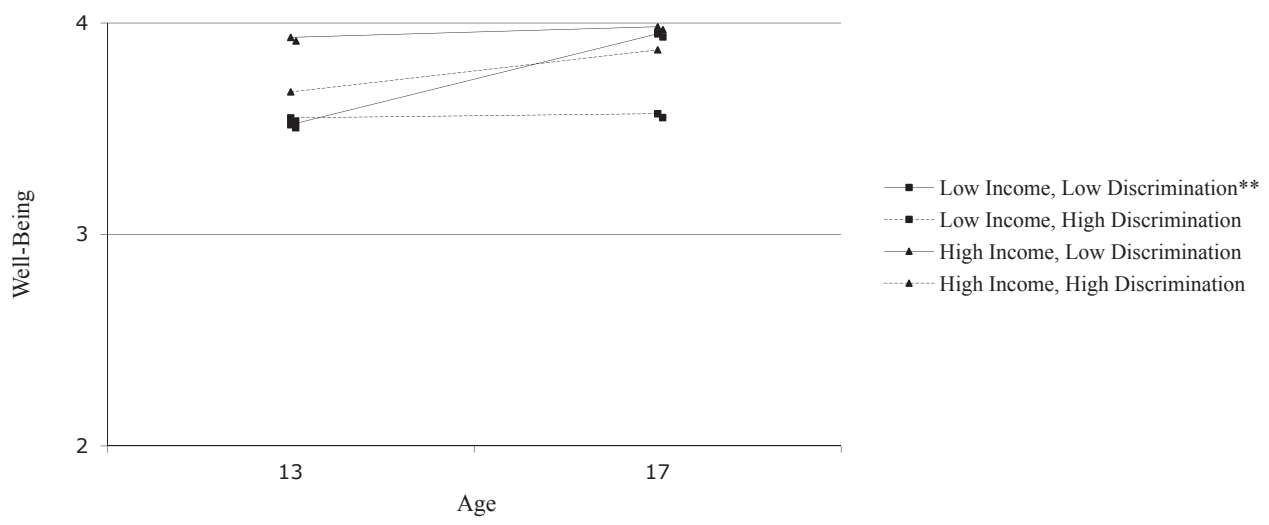

Figure 3. Three-way interaction between caregiver discrimination, income, and age on child well-being. $* * p<.01$.

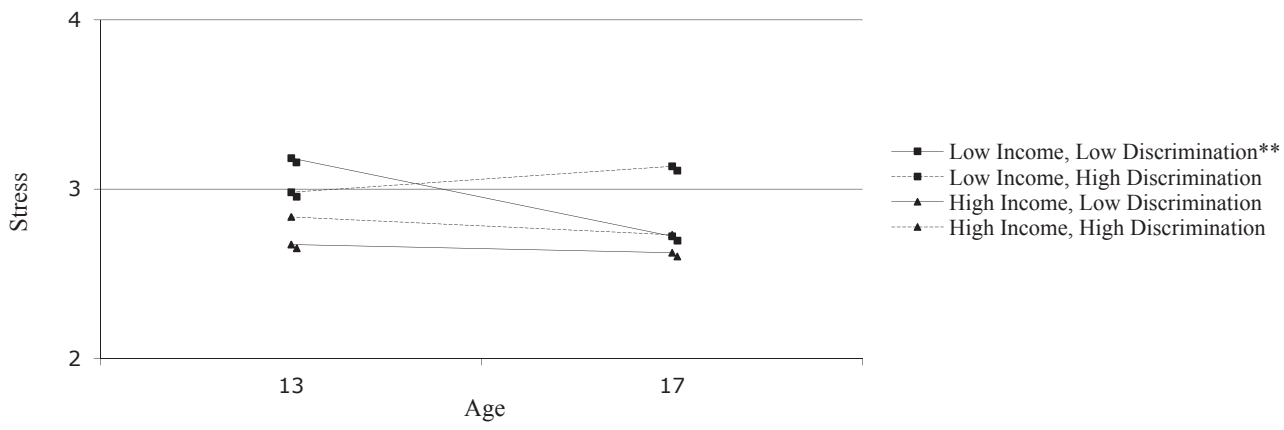

Figure 4. Three-way interaction between caregiver discrimination, income, and age on child stress. ${ }^{* *} p<.01$.

income-lower discrimination group had significantly higher levels of well-being than the higher incomehigher discrimination group, $\omega=-.16(.08), t=-2.04$, $p=.04$. By age 17 years, the two groups reported comparable levels of well-being, $\omega=-.07(.10), t=-.69$, $p=.49$. The lower income groups exhibited the opposite pattern. At age 13 years, the lower income-lower discrimination and lower income-higher discrimination groups reported similar levels of well-being, $\omega=.02(.07), t=0.30, p=.77$. At age 17 years, the lower income-lower discrimination group reported significantly higher levels of well-being than the lower income-higher discrimination group, $\omega=-.24(.10)$, $t=-2.34, p=.02$. In sum, the two higher income groups' reports of well-being converged over time. Conversely, the developmental trajectories of the lower income groups diverged. Whereas the lower income-lower discrimination group reported increased well-being over time, the lower incomehigher discrimination group maintained low levels of well-being over time, $t=-1.86, p=.07$.

Perceived stress. Finally, we found that caregiver discrimination and income moderated the trajectory of adolescents' perceived stress (column 3, Table 4;
Figure 4). We found a significant difference in slopes between the two lower income groups. When caregivers reported lower income and lower discrimination, adolescents reported relatively high levels of stress at age 13 years. By age 17 years, these adolescents' stress levels declined significantly, $\omega=-.12$ (.04), $t=-3.07, p=.002$. In contrast, when caregivers reported lower income and higher discrimination, adolescents reported relatively high levels of stress at age 13 years and maintained those high levels until age 17 years, $\omega=.04(.04), t=1.02, p=.31$.

Further testing showed that the relative positioning of the groups actually changed at ages 13 years and 17 years. At age 13 years, the lower incomelower discrimination group reported higher stress than the lower income-higher discrimination group, $\omega=-.12(.07), t=-1.95, p=.05$. By age 17 years, the lower income-lower discrimination group reported lower levels of stress than the lower income-higher discrimination group, $\omega=.26(.10)$, $t=2.68, p=.01$. In sum, adolescents in both lower income groups began the study with relatively high levels of stress. The lower income-lower discrimination group's stress declined over time, but the 
lower income-higher discrimination group did not decline, leading to a significant difference at age 17 years.

The simple slopes test indicated no significant change in stress levels for either of the higher income groups. Both the higher income-higher discrimination group and the higher income-lower discrimination group reported relatively low levels of perceived stress at age 13 and maintained low levels until age 17 years.

\section{Discussion}

Our findings partially support our hypotheses regarding the detrimental effects of caregivers' discrimination experiences on their adolescent children's psychological well-being. We found associations over time between caregivers' perceptions of discrimination and adolescents' symptoms of depression and psychological well-being. Specifically, we found that more caregiver experiences with discrimination predicted greater symptoms of depression and lower psychological well-being among adolescents. These findings are consistent with Coll et al.'s (1996) integrative theoretical model that proposes that the social positioning of ethnic minority youth influences their psychosocial development. Furthermore, these findings lend support to the notion that oppressive forces, such as discrimination, have the potential to alter family processes. In addition, these findings are consistent with those of other studies that have investigated associations between parent or caregiver experiences with discrimination and youth's psychosocial outcomes (Gibbons et al., 2004; Riina \& McHale, 2010). Although we do not know whether or not youth witnessed the discriminatory experiences their caregivers reported, our findings suggest that whether through direct or indirect exposure, caregivers' experiences with discrimination negatively affected African American adolescents' psychological functioning.

\section{Caregiver Discrimination and Adolescent Mental Health}

Bivariate correlations showed that caregiver discrimination was positively correlated with depressive symptoms, negatively correlated with well-being, and uncorrelated with perceived stress. These relations were largely the same in the growth curve models. There was a trend level association between caregiver discrimination and adolescent depressive symptoms, such that greater reports of caregiver discrimination were associated with elevated levels of adolescent depressive symptoms. Similarly, caregiver discrimination was negatively related to adolescent well-being. Of note, we did not find significant associations between caregivers' reported discrimination experiences and adolescents' perceived stress in either the bivariate correlation or in the more elaborate model. This null finding may be due to the fact that on average, adolescent participants reported elevated levels of perceived stress over time (average value of 3 on scale of $0-4$ ). Furthermore, there was limited variance in this outcome across waves and the reliability of the perceived stress items for our sample was less than ideal at each study wave. Given these measurement difficulties, the relation between perceived stress and caregiver discrimination may have been more difficult to find, relative to the other outcomes in this sample.

\section{Moderators of the Relation Between Caregiver Discrimination and Adolescent Mental Health}

Despite anticipating differential associations between caregiver's discrimination experiences and adolescents' psychological outcomes as a function of gender, we did not find gender differences in the associations between caregivers' discrimination experiences and adolescents' depressive symptoms or psychological well-being over time. We expected that girls would be more negatively affected by their caregivers' discrimination experiences than boys due to research findings that suggest that girls are more affected by interactions with their caregivers and more sensitive to intrafamilial stress (Gore et al., 1993). It is possible that the male and female adolescents in our study were equally sensitive to caregivers' experiences with discrimination. Direct exposure to this discriminatory treatment may have resulted in boys and girls having similar psychological reactions (i.e., increased depressive symptoms and decreased psychological well-being), which would be consistent with other study findings regarding decreased psychological well-being among African American youth who have witnessed acts of racial discrimination against other African Americans in their communities (Simons et al., 2002).

We also did not find moderation effects of family income on the association between caregiver's discrimination experiences and adolescents' psychological outcomes; however, we did find that family income interacted with caregivers' discrimination 
experiences and the linear growth term to explain adolescents' symptoms of depression, perceived stress, and psychological well-being over time. Notably, we found similar patterns of relations across all three outcome variables (i.e., adolescents' depressive symptoms, perceived stress, and psychological well-being).

Overall, adolescent participants from higher income families whose caregivers' reported fewer discrimination experiences reported the most positive psychological outcomes over time. During early adolescence, these participants displayed the most positive psychological profiles. Our findings suggest that these adolescents displayed minimal change in their psychological outcomes as they transitioned from middle to late adolescence.

In contrast, adolescent participants from lower income families whose caregivers' reported more discrimination experiences demonstrated the least positive psychological outcomes over time. In comparison to their peers, these youth reported comparable levels of psychological health during early adolescence, but across all outcomes these youth showed no improvements over time and demonstrated increases in perceived stress as they transitioned through adolescence. By late adolescence, these youth reported the most depressive symptoms and perceived stress and the lowest levels of psychological well-being.

Adolescent participants whose caregivers reported more discrimination experiences but who were from higher income families also reported comparable levels of psychological health during early adolescence, but these youth demonstrated improvements over time. Specifically, these youth reported reduced depressive symptoms as they moved through middle and into late adolescence. Adolescent participants from lower income families with caregivers who reported fewer discrimination experiences appeared to have the least healthy psychological profiles during early adolescence; however, similar to the previously described group, these youth demonstrated substantial improvements over time. These youth showed declines in depressive symptoms and perceived stress while also demonstrating increases in psychological well-being.

Taken together, our findings appear to illustrate processes of risk and resilience (Fergus \& Zimmerman, 2005) among African American adolescents. Consistent with notions of risk and resilience, youth experiencing the fewest risk factors in our study (i.e., those from higher income families and with caregivers' reporting less discrimination experiences) displayed the most positive mental health trajectories over time, and those exposed to the greatest amount of risk (i.e., lower family income and greater caregiver discrimination experiences) displayed the least positive mental health trajectories over time. Although higher levels of risk (i.e., experiencing lower family income and higher caregiver discrimination experiences) may have had deleterious consequences, our findings suggest that youth may have been able to cope effectively with more moderate levels of risk. Thus, youth were able to successfully manage and cope with the presence of one risk factor (i.e., higher caregiver discrimination experiences or lower family income) over time. Some models of resilience (i.e., challenge and inoculation models) suggest that youth may be more able to employ resources and cope with moderate levels of risk, whereas exposure to higher levels of risk may be nearly impossible to overcome (Zimmerman \& Arunkumar, 1994).

In addition to illustrating processes of risk and resilience among African American youth, our findings also suggest that higher family income may help buffer African American youth from the negative effects of caregiver discrimination experiences on their mental health over time. During early adolescence, higher caregiver discrimination experiences appeared to exert a negative effect on adolescents' psychological outcomes regardless of youth's family income level. Yet, over time, youth from higher income families appeared to be less negatively impacted by their caregivers' discrimination experiences, whereas youth from lower income families maintained less positive psychological profiles over time. This finding suggests that African American adolescents from higher income families may have increased access to resources that can help promote positive adaptation in the face of risk.

Another possibility is that more affluent African American caregivers and adolescents may rely on their higher income status to protect them from the negative effects of racial discrimination. Lacy and Harris (2008) acknowledge the historic association between Blackness and poverty and suggest that material wealth may afford African Americans opportunities to reject the stigma of poverty that is associated with their race and as a consequence, be better positioned to grapple with discrimination experiences targeted at them or their loved ones. Given that discriminatory experiences communicate messages of inferiority and that U.S. society is materialistic and status conscious, it seems plausible that more affluent African Americans may have increased opportunities to reject messages of racial 
inferiority in light of the social status that their financial success may provide. Thus, higher income participants in the current study may have fared better over time due to their ability, at least in part, to reject negative stereotypes about their racial group conveyed through caregivers' discrimination experiences.

\section{Study Strengths and Limitations}

This study adds to a body of research demonstrating the deleterious effects of caregivers' experiences with racial discrimination on adolescents' psychological functioning (e.g., Gibbons et al., 2004). Moreover, the study incorporated a longitudinal approach, which allowed us to explore the ways in which the relation between caregivers' discrimination experiences and adolescents' psychological functioning changed over time. First, because of the broad age range at the first wave of data collection (ages 12-17) and because the study included up to three waves of data for each participant, we were also able to examine the relation between individual-level development and caregiver discrimination in middle and late adolescence. Second, we examined three indicators of psychological health to explore how discrimination affected a range of important domains. We found that the relation between the development of adolescents' mental health and caregiver discrimination varied as a function of family income. The findings were similar across three domains of psychological functioning, consistently demonstrating that the lower income-higher caregiver discrimination group had the poorest outcomes over time. Third, the study also benefited from substantial income variation in the sample. Although the community sampled for the study was relatively affluent and well educated, there was still substantial diversity in household income across families in the sample. This variation helped us to uncover the complex interaction between caregiver discrimination and adolescents' psychological development as a function of caregiver income. It is also important to note that preliminary analyses indicated that caregiver discrimination was not a proxy for adolescents' personal experiences with discrimination. We found a low correlation between caregiver discrimination and children's experiences of discrimination $(r=.14, p<.01)$ indicating that parent and child discrimination experiences were separate constructs. This finding underscores the need for continued evaluation of the potential deleterious impact of caregiver discrimination on child mental health.
Although we believe this study is a novel and valuable addition to a growing literature, there are some limitations. Our assessment of the relation between caregiver discrimination and adolescent psychological functioning was contemporaneous; thus, we cannot be certain of the causal direction. It is possible that caregiver discrimination experiences were a primary or secondary determinant of adolescents' psychological functioning. However, because discrimination and functioning were measured at the same time, we cannot assume that caregiver discrimination caused the changes in psychological functioning. In addition, the interpretation of findings is limited by the study design. Although the accelerated longitudinal design allowed us to test the model as if we had data from each participant for the entirety of the study period, we did not have data from each participant across all years. Complete longitudinal data from each participant across the age range is the gold standard for developmental analysis. With complete data across the age range, we could be more confident that we accurately modeled the trajectory of each outcome variable in the sample.

\section{Conclusions and Directions for Future Research}

Taken together, our findings show that racial discrimination may have far-reaching implications for African American families. This research highlights the ways in which family income may either mitigate or exacerbate the detrimental impact of caregivers' experiences of racial discrimination on adolescent mental health. The impact of discrimination and family income changed as adolescents moved from early to middle adolescence. These findings hint at a complex interplay of social position, discrimination, and psychological development.

Additional research should consider the family dynamics that underlie this relation. One possible avenue of exploration involves the ways in which aspects of the caregiver-child relationship might affect the association between caregiver discrimination experiences and child mental health. In the present study, we examined whether this association differed in mother-adolescent dyads as opposed to other family relationships (e.g., fatheradolescent or grandmother-adolescent). Although we did not find that the association differed, the low number of adolescents in the sample who were paired with fathers, grandparents, or other nonmaternal caregivers limited the power of our analyses to detect such differences. Future studies 
with a greater diversity and a higher number of adolescent-caregiver pairings would help to further illuminate these findings. Furthermore, the quality of the relationship between caregivers and children (e.g., their level of attachment, their closeness to one another, or the extent to which they talk with one another) could also affect the degree to which caregiver discrimination relates to child mental health. Future research that includes assessments of caregiver-child relationship quality and examines multiple pathways from caregiver discrimination to child mental health will build on the current study and advance our understanding of the ways in which caregiver discrimination may detrimentally affect child mental health.

\section{References}

Aboud, F. E. (1989). Children and prejudice. Hoboken, NJ: Blackwell.

Arnett, J. J. (1999). Adolescent storm and stress, reconsidered. American Psychologist, 54, 317-326. http://dx.doi. org/10.1037/0003-066X.54.5.317

Bell, R. Q. (1953). Convergence: An accelerated longitudinal approach. Child Development, 24, 145-152. http:// dx.doi.org/10.2307/1126345

Bell, R. Q. (1954). An experimental test of the accelerated longitudinal approach. Child Development, 25, 281-286. http: / / dx.doi.org/10.2307/1126058

Brody, G. H., Chen, Y., Murry, V. M., Ge, X., Simons, R. L., Gibbons, F. X., et al. (2006). Perceived discrimination and the adjustment of African American youths: A five-year longitudinal analysis with contextual moderation effects. Child Development, 77, 1170-1189. http:// dx.doi.org/10.1111/j.1467-8624.2006.00927.x

Broman, C. L. (1997). Race-related factors and life satisfaction among African Americans. Journal of Black Psychology, 23, 36-49. http://dx.doi.org/10.1177/00957984970 231004

Caughy, M. O., O'Campo, P. J., \& Muntaner, C. (2004). Experiences of racism among African American parents and the mental health of their preschool-aged children. American Journal of Public Health, 94, 2118-2124. http:// dx.doi.org/10.2105/AJPH.94.12.2118

Clark, R., Anderson, N. B., Clark, V. R., \& Williams, D. R. (1999). Racism as a stressor for African Americans: A biopsychosocial model. American Psychologist, 54, 805816. http:/ /dx.doi.org/10.1037/0003-066X.54.10.805

Cohen, S., Kamarck, T., \& Mermelstein, R. (1983). A global measure of perceived stress. Journal of Health and Social Behavior, 24, 385-396.

Coll, C. G., Crnic, K., Lamberty, G., Wasik, B. H., Jenkins, R., García, H. V., et al. (1996). An integrative model for the study of developmental competencies in minority children. Child Development, 67, 1891-1914. http://dx. doi.org/10.2307/1131600
Cross, W. E., Jr., \& Fhagen-Smith, P. (2001). A life-span developmental model of racial identity. In C. J. Wijeyesinghe \& B. W. Jackson (Eds.), New perspectives on racial identity development: A theoretical and practical anthology (pp. 243-270). New York: New York University Press

Dawson, J. F., \& Richter, A. W. (2006). Probing three-way interactions in moderated multiple regression: Development and application of a slope difference test. Journal of Applied Psychology, 91, 917-926. http://dx.doi.org/ 10.1037/0021-9010.91.4.917

Fergus, S., \& Zimmerman, M. A. (2005). Adolescent resilience: A framework for understanding healthy development in the face of risk. Annual Review of Public Health, 26, 399-419.

Fisher, C. B., Wallace, S. A., \& Fenton, R. E. (2000). Discrimination distress during adolescence. Journal of Youth and Adolescence, 29, 679-695. http://dx.doi.org/ 10.1023/A:1026455906512

Fitzmaurice, G. M., Laird, N. M., \& Ware, J. H. (2004). Applied longitudinal analysis. New York: Wiley.

Gibbons, F. X., Gerrard, M., Cleveland, M. J., Wills, T. A., \& Brody, G. (2004). Perceived discrimination and substance use in African American parents and their children: A panel study. Journal of Personality and Social Psychology, 86, 517-529. http://dx.doi.org/10.1037/ 0022-3514.86.4.517

Gore, S., Aseltine, R. H., Jr., \& Colten, M. E. (1993). Gender, social-relationship involvement, and depression. Journal of Research on Adolescence, 3, 101-125. http://dx. doi.org/10.1207/s15327795jra0302_1

Greene, M. L., Way, N., \& Pahl, K. (2006). Trajectories of perceived adult and peer discrimination among Black, Latino, and Asian American adolescents: Patterns and psychological correlates. Developmental Psychology, 42, 218-238. http://dx.doi.org/10.1037/ 0012-1649.42.2.218

Harrell, S. (1997). Development and initial validation of scales to measure racism-related stress. Poster presented at the 6th biennial conference on Community Research and Action, Society for Community Research and Action, Columbia, SC.

Hox, J. J. (2002). Multilevel analysis. Techniques and applications, Mahwah, NJ: Erlbaum.

Hughes, D., \& Chen, L. (1997). When and what parents tell children about race: An examination of race-related socialization among African American families. Applied Developmental Science, 1, 200-214. http://dx.doi.org/ $10.1207 /$ s1532480xads0104_4

Jackson, J. S., Brown, T. N., Williams, D. R., Torres, M., Sellers, S. L., \& Brown, K. (1996). Racism and the physical and mental health status of African Americans: A thirteen year national panel study. Ethnicity $\mathcal{E}$ Disease, 6, 132-147.

Johnson, D. J. (2001). Parental characteristics, racial stress, and racial socialization processes as predictors of racial coping in middle childhood In A. M. Barnett \& J. M. Contreras (Eds.), Forging links: African American children clinical development perspectives (pp. 57-74). Westport, CT: Praeger. 
Kessler, R. C., Mickelson, K. D., \& Williams, D. R. (1999). The prevalence, distribution, and mental health correlates of perceived discrimination in the United States. Journal of Health and Social Behavior, 40, 208-230. http:/ / dx.doi.org/10.2307/2676349

Lacy, K., \& Harris, A. L. (2008). Breaking the class monolith: Understanding class differences in Black adolescents' attachment to racial identity. In A. Lareau \& D. Conley (Eds.) Social class: How does it work (pp. 152178). New York: Russell Sage Foundation.

McArdle, J. J., Ferrer-Caja, E., Hamagami, F., \& Woodcock, R. W. (2002). Comparative longitudinal structural analyses of the growth and decline of multiple intellectual abilities over the life-span. Developmental Psychology, 38, 115-142. http://dx.doi.org/10.1037/0012-1649.38.1. 115

McHale, S. M., Crouter, A. C., Kim, J., Burton, L. M., Davis, K. D., Dotterer, A. M., et al. (2006). Mothers' and fathers' racial socialization in African American families: Implications for Youth. Child Development, 77, 1387-1402. http: / /dx.doi.org/10.1111/j.1467-8624.2006.00942.x

Meyer, I. H. (2003). Prejudice as stress: Conceptual and measurement problems. American Journal of Public Health, 93, 262-265. http: / /dx.doi.org/10.2105/AJPH.93.2.262

Miyazaki, Y., \& Raudenbush, S. W. (2000). Tests for linkage of multiple cohorts in an accelerated longitudinal design. Psychological Methods, 5, 44-63. http://dx.doi. org/10.1037/1082-989X.5.1.44

Preacher, K. J., Curran, P. J., \& Bauer, D. J. (2006). Computational tools for probing interactions in multiple linear regression, multilevel modeling, and latent curve analysis. Journal of Educational and Behavioral Statistics, 31, 437. http:/ / dx.doi.org/10.3102/10769986031004437

Quintana, S. M. (1998). Children's developmental understanding of ethnicity and race. Applied and Preventive Psychology, 7, 27-45. http://dx.doi.org/10.1016/S09621849(98)80020-6

Radloff, L. S. (1977). The CES-D scale: A self-report depression scale for research in the general. Applied Psychological Measurement, 1, 385-401. http://dx.doi. org/10.1177/014662167700100306

Raudenbush, S. W., \& Bryk, A. S. (2002). Hierarchical linear models: applications and data analysis methods. Thousand Oaks, CA: Sage.

Raudenbush, S. W., \& Chan, W. S. (1992). Growth curve analysis in accelerated longitudinal designs. Journal of Research in Crime and Delinquency, 29, 387-411. http:// dx.doi.org/10.1177/0022427892029004001
Riina, E. M., \& McHale, S. M. (2010). Parents' experiences of discrimination and family relationship qualities: The role of gender. Family Relations, 59, 283-296. http:/ /dx. doi.org/10.1111/j.1741-3729.2010.00602.x

Rosenbloom, S. R., \& Way, N. (2004). Experiences of discrimination among African American, Asian American, and Latino adolescents in an Urban High School. Youth $\mathcal{E}$ Society, 35, 420-451. http://dx.doi.org/ $10.1177 / 0044118$ X03261479

Ryff, C. D. (1989). Happiness is everything, or is it? Explorations on the meaning of psychological well-being. Journal of Personality and Social psychology, 57, 1069-1081. http:/ /dx.doi.org/10.1037/0022-3514.57.6.1069

Seaton, E. K., Caldwell, C. H., Sellers, R. M., \& Jackson, J. S. (2008). The prevalence of perceived discrimination among African American and Caribbean Black youth. Developmental Psychology, 44, 1288-1297. http://dx.doi. org/10.1037/a0012747

Seaton, E. K., Caldwell, C. H., Sellers, R. M., \& Jackson, J. S. (2010). An intersectional approach for understanding perceived discrimination and psychological well-being among African American and Caribbean Black youth. Developmental Psychology, 46, 1372-1379. http://dx.doi. org/10.1037/a0019869

Seaton, E. K., Yip, T., \& Sellers, R. M. (2009). A longitudinal examination of racial identity and racial discrimination among African American adolescents. Child Development, 80, 406-417. http://dx.doi.org/10.1111/ j.1467-8624.2009.01268.x

Simons, R. L., Murry, V., McLoyd, V., Lin, K. H., Cutrona, C., \& Conger, R. D. (2002). Discrimination, crime, ethnic identity, and parenting as correlates of depressive symptoms among African American children: A multilevel analysis. Development and Psychopathology, 14, 371-393. http://dx.doi.org/10.1017/ S0954579402002109

Williams, D. R., Neighbors, H. W., \& Jackson, J. S. (2008). Racial/ethnic discrimination and health: Findings from community studies. American Journal of Public Health, 98, S29-S37.

Wong, C. A., Eccles, J. S., \& Sameroff, A. (2003). The influence of ethnic discrimination and ethnic identification on African American adolescents' school and socioemotional adjustment. Journal of Personality, 71, 1197-1232. http:// dx.doi.org/10.1111/1467-6494.7106012

Zimmerman, M. A., \& Arunkumar, R. (1994). Resiliency research: Implications for schools and policy. Social Policy Report, 8, 1-17. 